\title{
An Adaptive Fault Locator System For Transmission Lines
}

\author{
Joe-Air Jiang Ying-Hong Lin Chih-Wen Liu Jun-Zhe Yang Tong-Ming Too \\ Member, IEEE
}

\author{
Department of Electrical Engineering \\ National Taiwan University, Taipei, Taiwan
}

\begin{abstract}
This paper presents an adaptive fault locator system which utilizes two-terminal Global Positioning System (GPS) based synchronized phasor measurements to on-line estimate line parameters in steady-state and calculate fault location in the occurrence of faults. Uncertainty about the line parameters, which usually results in a significant error in the calculated fault location, can be resolved in the proposed parameter estimation algorithm. A novel fault location index in terms of Clarke components of the synchronized voltage and current phasors is also proposed to calculate the fault location in the accuracy of up to $99.9 \%$. This paper also proposes a new Discrete Fourier Transform (termed as Smart Discrete Fourier Transform, SDFT) based algorithm to eliminate system noise and measurement errors. The field tests of two GPS based phasor measurement prototypes demonstrate that a synchronization error between two remotely spaced prototypes is small enough for fault locator. The performance of the parameter estimation algorithm and index is illustrated with simulation results from EMTP.
\end{abstract}

Keywords: Fault Detection/Location Index, Parameter Estimation Algorithm, Discrete Fourier Transforms (DFT), Phasor Measurement Unit (PMU).

\section{INTRODUCTION}

In power systems, high voltage transmission and distribution lines are vital links that achieve the essential continuity of service from the generating plants to the end users. Fault location on transmission lines is thus a very well known and important problem that has been studied for a long time. From views of economics and quality of power feeding, the importance of fault location of transmission lines is increasingly. The more accuracy of fault location has been obtained the easier task for inspection, maintenance, and repair of the line we can achieve. Rapid restoration of service could reduces customer complaints, outage time, loss of revenue, and crew repair expense.

In the past, the most common approach was to model transmission lines using phasors. [1-9]. This approach is referred to as impedance based measurement technique, and is classified into two methods. The earlier developed one is one-terminal data method, and the other is currently more prevalent one so-called two-terminal method. These methods use voltage and current phasors to determine the impedance to the fault location, and both suffers from errors mentioned in many papers [10-15]. A number of factors influence the sensitivity of fault location algorithm for protection of a specific transmission line. Among the investigations [1-15] developed at early stage, the accuracy of those proposed algorithms are affected with at least one of the following factors:

(i) Parameter uncertainty due to line aging, line asymmetry and environmental factors.

(ii)System frequency floating due to severely variation in power flow.

(iii) Inadequate modeling of transmission lines.

(iv) Synchronization errors due to measurements of two- terminal data.

(v)Measurement errors due to hardware, e.g. phase shift error involved in CCVTs.
All of these factors will be taken into account and solved by the algorithms proposed in this paper.

The paper is organized into six sections, the first of which is introduction. In the second section, we introduce the proposed algorithms utilized in the adaptive fault locator system, including brief derivation of fault location index, the parameter estimation technique, and a new Discrete Fourier Transform based algorithm (SDFT). In the third section, the implementation of a GPS based Phasor Measurement Unit (PMU) is presented. Those algorithms mentioned in the second section are combined with PMU configuration to form the adaptive fault locator system and will be described in section IV. The reliability of proposed parameter estimation technique has been verified through the field test. The performances of proposed fault location algorithm for various faults are demonstrated with the data generated by EMTP simulator in the fifth section. Finally, section VI is devoted to conclusions.

\section{Fault Location Index}

\section{THE PROPOSED ALGORITHMS}

\section{Single-phase Case:}

In Fig. 1, suppose that a midway fault occurred at the point $F$ which is $x=D L \mathrm{~km}$ away from receiving end $\mathrm{R}$. $\mathrm{L}$ is the total length of the transmission line, and $D$ is the per unit distance from receiving end to the fault. $D$ is also used as a fault location index. In case of a fault occurrence at the point $F$, the transmission line is thus divided into two parts. These two homogeneous line sections, SF and FR, still can be regarded as perfect transmission lines. We can visualize that there is a pre-fault transmission line as shown in Fig.1. If we put the two sets of measured data $\left(V_{s}, I_{s}\right)$ and $\left(V_{R}, I_{R}\right)$ on the same reference position $x=0$ and take into account the boundary condition on both ends, then the voltage on the fault position $x=D L$ $\mathrm{km}$ can be expressed as:

$$
\begin{aligned}
& V_{F}=\frac{1}{2 \exp (\gamma L)}\left(V_{S}+Z_{C} I_{S}\right) \exp (\gamma D L)+\frac{1}{2} \exp (\gamma L)\left(V_{S}-Z_{C} I_{S}\right) \exp (-\gamma D L) \\
& V_{F}=\frac{V_{R}+Z_{C} I_{R}}{2} \exp (\gamma D L)+\frac{V_{R}-Z_{C} I_{R}}{2} \exp (-\gamma D L)
\end{aligned}
$$

Since $V_{p}$ is the voltage at the same point $\mathrm{F}$, equating the Eq.(1) and Eq. (2) and solving the fault detection/location index $D$ yields:

$$
D=\ln (N / M) / 2 \gamma L
$$

where

$$
N=\frac{V_{R}-Z_{C} I_{R}}{2}-\frac{V_{S}-Z_{C} I_{S}}{2} \exp (\gamma L)
$$

and

$$
M=\frac{V_{S}+Z_{C} I_{S}}{2} \exp (-\gamma L)-\frac{V_{R}+Z_{C} I_{R}}{2}
$$

It is worthy to note that no assumption in the procedure of derivation for the fault location index $D$ is made. Hence, the index $D$ is very robust.

\section{Three-phase Case}

Consider an arbitrary three-phase transmission line illustrated in Fig.2, which consists of phase a, b, c and a ground system consisting of earth and ground wires. We can utilize the Clarke transformation [12] to solve the coupling effect of the inter-phase. As same treatment with single-phase case, we could still take the voltages at fault point $\mathrm{F}$ which locate at $x=D L \mathrm{~km}$ away from receiving end and $x=0$ as boundary condition, the fault location index are therefore solved as

$$
D_{i}=\frac{\ln \{[A(i)-C(i)] /[E(i)-B(i)]\}}{2 \Gamma(i, i) L} \quad i \equiv 1,2,3
$$




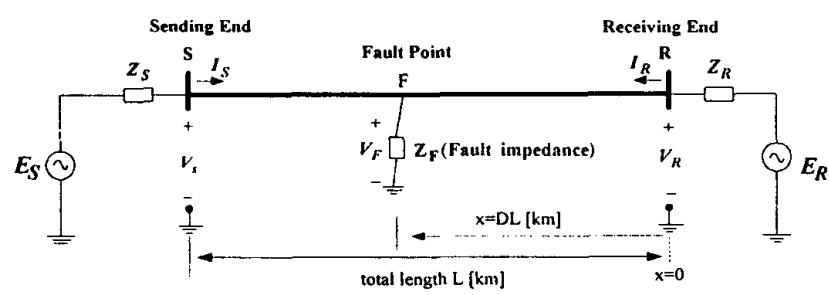

Fig. 1 A single-phase faulted transmisston line.

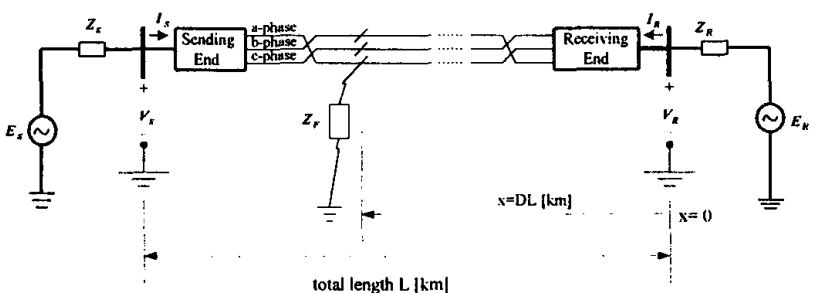

Fig. 2 A three-phase transposed transmission line

$$
\begin{aligned}
& A(i)=\frac{1}{2}\left[V_{R m}(i)+Z_{C}(i) I_{R m}(i)\right] \\
& B(i)=\frac{1}{2}\left[V_{R m}(i)-Z_{C}(i) I_{R m}(i)\right] \quad i \equiv 1,2,3 \\
& C(i)=\frac{1}{2} \exp \left[-\Gamma(i, i) L\left[V_{S m}(i)+Z_{C}(i) I_{S m}(i)\right]\right. \\
& E(i)=\frac{1}{2} \exp \left[-\Gamma(i, i) L\left[V_{S m}(i)-Z_{C}(i) I_{S m}(i)\right]\right.
\end{aligned}
$$

where $I(i, i)$ and $Z_{\mathrm{c}}(i, i)$ respectively represent the diagonal entries of $3 \times 3$ modal propagation constant matrix and surge impedance matrix, and subscripts $S$ and $R$ represent the sending and receiving ends of the transmission line. It should be noted that the quantities utilized in Eq.(7) could also be pure-fault data. Therefore, the effects of line loading on the accuracy of the index can be eliminated.

\section{On-line Parameter Estimation Algorithm}

Almost all of the existing fault location techniques did not consider uncertainty about the line parameters. With the advent of synchronized phasor measurement technique, one can obtain extremely accurate phasors. Consider the three-phase transposed transmission line shown in Fig.2, using the technique proposed, we can on-line monitor the status of transmission line, and extract the true phasor measurement data $\left(V_{S}, I_{S}\right)$ and $\left(V_{R}, I_{R}\right)$ from both line ends by the SDFT filtering approach. These data can be utilized to derive the characteristic impedance and propagation constant of transmission lines. Such line parameters thus can be respectively expressed as following:

$$
Z_{C m}=\left[\frac{V_{R m}^{2}-V_{S m}^{2}}{I_{S m}^{2}+I_{R m}^{2}}\right]^{1 / 2} \quad m=1,2,3
$$

and

$$
\gamma_{m}=\cosh ^{-1}\left(K_{m}\right) / L \quad m=I, 2,3
$$

where

$$
K_{m}=\frac{V_{S m} I_{S m}+V_{R m} I_{R m}}{V_{S m} I_{R m}+V_{R m} I_{S m}} \quad m=1,2,3
$$

The entire quantities mentioned above all are the modal components of measured signals obtained by Clarke transformation, where subscript $m=1,2,3$ denote $0, \alpha, \beta$-mode, respectively. It should be noted that in order to avoid zero-mode effects and uncertainties in zero-mode parameters, the zero-mode calculation is not recommended. It has been verified in field-test that the proposed on-line parameter estimation technique is extremely accurate for high voltage distribution lines. Such a parameter estimation technique can also be suitable for EHV/UHV transposed transmission case

\section{The SDFT Algorithm}

The procedure of the SDFT's derivation is similar to that of DFT. Limiting to the space of paper presented, we will just describe here the derivation of the SDFT briefly. The readers are encouraged to refer the paper [16] for the detail description of the SDFT. Consider a sinusoidal signal of frequency $\omega=2 \pi(60+\Delta f)$, given by:

$$
x(t)=X \cos (\omega t+\phi)
$$

where $\mathrm{x}$ : the amplitude of the voltage/current signal, $\phi$ : the phase angle of the voltage/current signal, $\Delta f:$ the frequency deviation from the fundamental frequency.

Taking frequency deviation $(\omega=2 \pi(60+\Delta f)$ ) into consideration, after some algebraic manipulations, the sampled components of the new DFT of sample set $\{x(\mathrm{k})\}$ is given by:

$\tilde{x}_{r}=A_{r}+B_{r}$

where $A_{r}$ and $B_{r}$ are defined as:

$A_{r}=\frac{\bar{x}}{N} \frac{\sin \left(N \theta_{1} 2\right)}{\sin \left(\theta_{1} 2\right)} \exp \left\{j \frac{\pi}{60 N}[\Delta f(2 r+N-1)+120 r]\right\}$

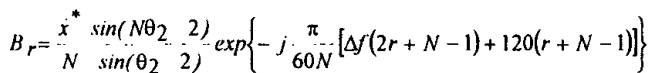

$\theta_{1}=\frac{2 \pi \Delta /}{60 N}$

$$
\theta_{2}=-\frac{2 \pi\left(2+\frac{\Delta r}{60}\right)}{N}
$$

$N=$ sampling rate $/ 60$

$\bar{x}$ is the phasor of signal $x(t)$, and '*' denotes complex conjugate.

In the DFT method, it assumes that the frequency deviation is small enough to be ignored, and it is always considers $\hat{x} \approx A_{r}$, so the traditional DFT based algorithm would incur errors in estimating frequency and phasor when frequency deviates from nominal frequency $(60 \mathrm{~Hz})$. If we want to get the exact solution, we must take $B_{r}$ into consideration. So we define:

$$
a=\exp \left[j \frac{\pi}{60}(2 \Delta f+120)\right]
$$

After some algebraic manipulations, the exact solution of the frequency can be obtained to expressed as:

$$
f=60+\Delta f=\cos ^{-1}[\operatorname{Re}(a)]^{*} \frac{60 N}{2 \pi}
$$

where $a$ satisfies the quadratic equation shown in the followings:

$$
\hat{x}_{r+1} * a^{2}-\left(\hat{x}_{r}+\hat{x}_{r+2}\right) * a+\hat{x}_{r+1}=0
$$

Moreover, we can estimate phasor after getting exact ' $f$ ' by the following equations:

$$
\begin{aligned}
& A_{r}=\frac{\dot{x}_{r+1}^{*} a-\hat{x}_{r}}{a^{2}-1} \\
& x=a b s\left(A_{r}\right) \frac{N^{*} \sin (\pi \Delta f / 60 N)}{\sin (\pi \Delta f / 60)} \\
& \phi=\operatorname{angle}\left(A_{r}\right)-\frac{\pi}{60 N}[\Delta f \times(N-1)]
\end{aligned}
$$

The SDFT can smartly avoid the errors that arise when frequency deviates from the fundamental frequency and keep all the advantages of the DFT e.g., immune to harmonics of fundamental frequency, obtaining easily the parameters of amplitude and phase, and even the recursive computing can be used in the SDFT. These make the SDFT more accurate than the conventional DFT based techniques. In addition, this method is recursive and very easy to implement, so it is very suitable for use in real-time measurements.

\section{AN ADAPTIVE PMU BASED FAULT DETECTION/LOCATION TECHNIQUE}

The SDFT based method, line parameter estimation algorithm and fault location index are combined with PMU configuration to form the adaptive fault locator system. The overall diagram of the 


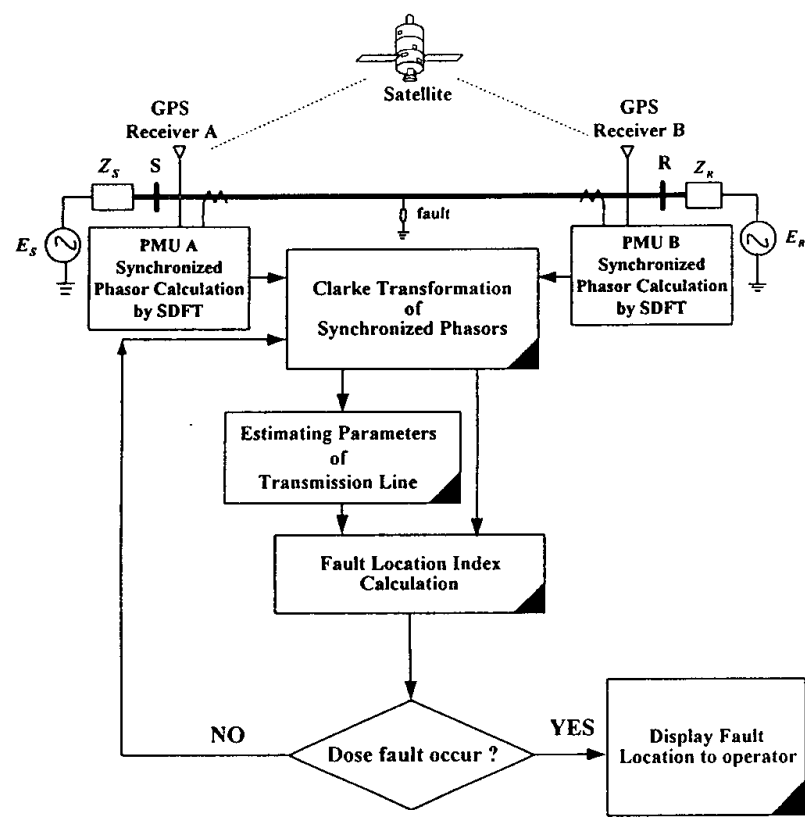

Fig.3 Configuration of adaptive PMU based fault detection/location system for EHV/UHV transmission line.

adaptive PMU based fault location technique is shown in Fig.3. A new developed SDFT algorithm has been built in the PMUs and can be utilized to process the data measured at both ends. Since the 'Global Synchronism Clock Generator (GSCG)' has also been equipped in PMU to provide an extremely accurate and reliable external reference clock signal, it can guarantee sampling synchronization to an accuracy of better than $1 \mu$-sec. The performance of the proposed PMU unit has been on-line demonstrated very well in the $161 \mathrm{kV}$ substations of Taipower system. The proposed algorithms are tested with the data generated from EMTP.

In order to evaluate the performance of the proposed fault location technique, the EMTP simulated voltage and current waveforms were directly taken as the synchronized sampled data (voltages and currents) from substations. The simulation examples are intentionally chosen as same with a practical $345 \mathrm{kV}$ transmission line of Taipower system. In order to truly include the errors involved in the measurement procedure of phasors, the Gaussian-type [14] noise signals were added into the EMTP generated data before they are processed in the algorithm. Next, the SDFT method is used to extract close-in fundamental phasors. These phasors are then transformed by Clarke transformation. The estimation of line parameters is proceeded in the next step. At this stage, the technique has generated the correct modal components that can be utilized to calculate the index. This technique possesses both abilities for detecting and locating the fault of transmission line. It is worthy to note that the proposed technique is recursive. Hence, the proposed technique is very suitable for the on-line monitoring applications.

\section{IMPLEMENTATION OF PHASOR MEASUREMENT UNIT}

\section{PMU and GSCG Configurations}

In this paper, we proposed an extremely accurate configuration for measuring phasors by virtue of Global Positioning System (GPS) and Phasor Measurement Units (PMU) technique, whose configuration is illustrated in Fig.4-(a). Due to availability of time synchronization in GPS, synchronized phasor measurement units based on GPS can real-time measured voltage and current phasors at

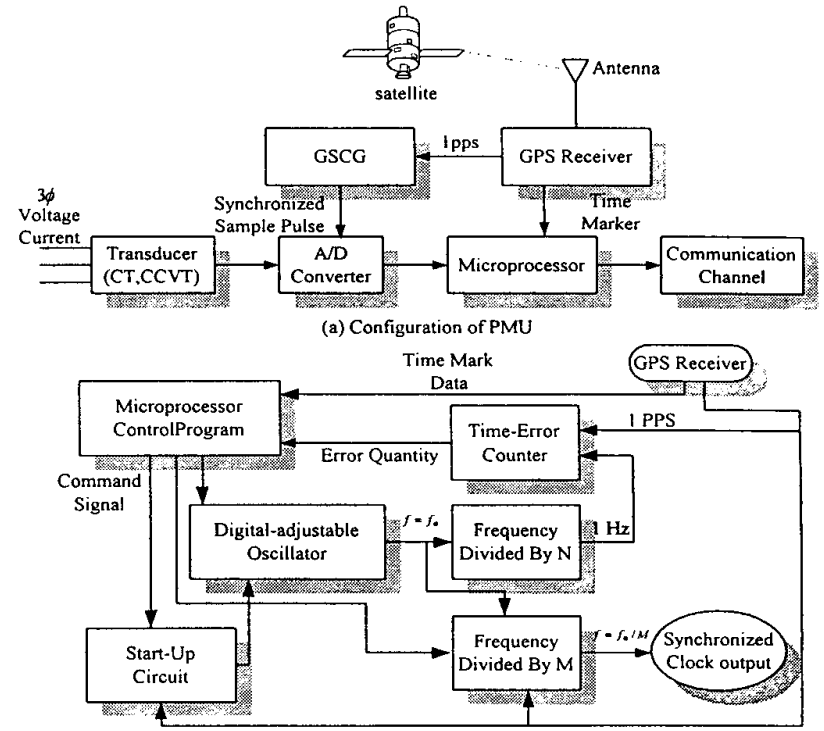

(b) Block Diagram of Global Synchronism Clock Generator (GSCG).

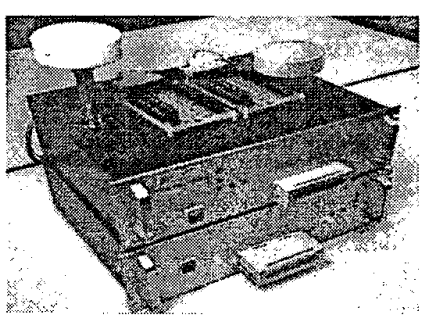

(c) Actual Implementation of GSCG

Fig. 4 The configuration of phasor measurement unit based on GPS and its actual implementation.

both bus ends of a transmission line. In order to reduce settling time, frequency jitter, and steady-state errors, we redesign a new timing device named "Global Synchronism Clock Generator (GSCG)" whose time error could remain within that of satellite's receiver. The block diagram and actual implementation of GSCG are shown in Fig.4-(b) and 4-(c) respectively. The very low cost for constructing the GSCG is one of several merits of our design. For example, the crystal oscillator utilized in our GSCG costs merely 28 NT\$ (about 1 US\$). Moreover, another important advantage of GSCG is its ability to automatically compensate for un-synchronization error by software built into the microprocessor. The frequency drift of GSCG can be controlled to within $\pm 0.1 \mathrm{PPM}$ and the rising edge error of two GSCG's clock $\Delta \mathrm{T}$ can remain within $1 \mu$ s (equivalent to $0.0126^{\circ}$ phase difference at $60 \mathrm{~Hz}$ ). The operation functions of PMU and GSCG are depicted as flow charts shown in Figs.4-(a) and (b), respectively.

\section{Field Test in $161 \mathrm{kV}$ Substations}

At the GPS system time 1998/4/22 02:57:00, the performance of PMU-GSCG has been verified with the field measurements which were performed at a single $161 \mathrm{kV}$ transmission line between Chung-Jeng and Chang-Te substations of Taipower system. The data measured at Chang-Te substation (receiving end) was substituted into the $\pi$-model transmission matrix of the line to calculate the theoretical values of voltage and current at Chung-Jeng (sending end). These three data are plotted in Fig.5 simultaneously for comparison. Fig.5-(a) only shows the c-phase current per unit value of transmission line, where we can observe that there are only about $2.5 \%$ difference between measured values and theoretical values at Chung - Jeng substation. The measured and theoretical phase angle 


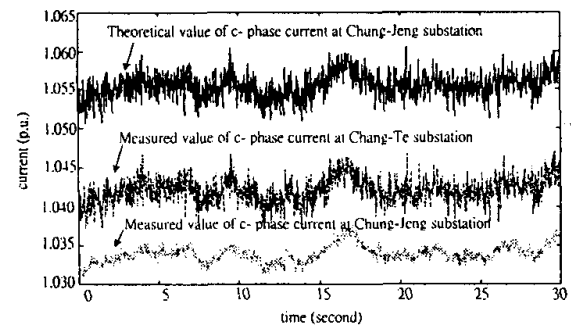

(a)
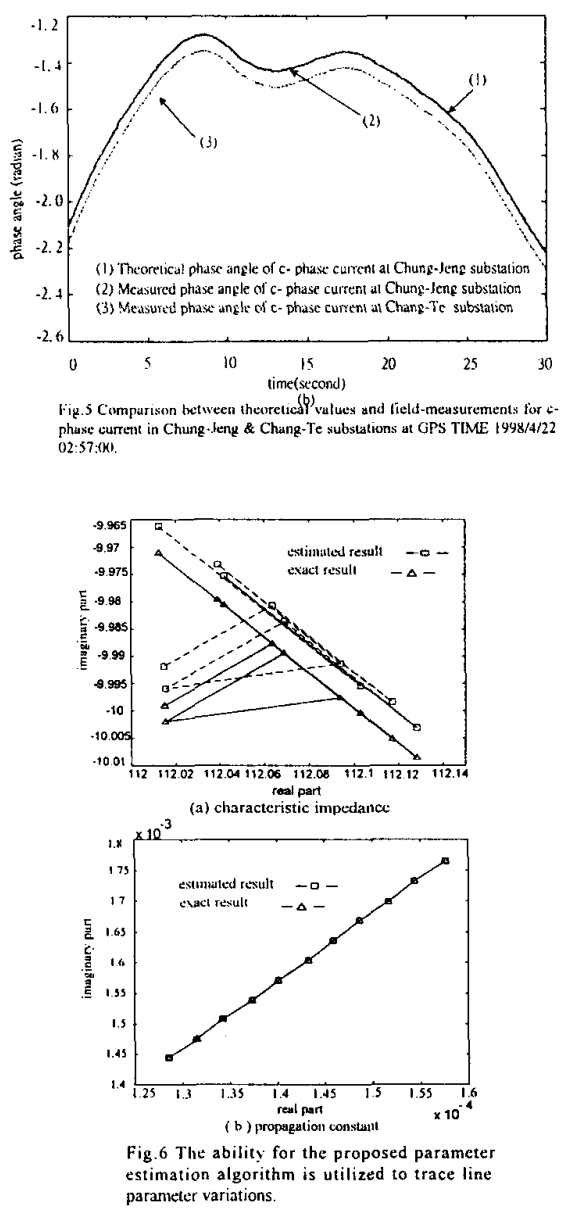

values of c-phase current of transmission line are shown in Fig.5-(b). It can be clearly seen that the phase difference between both data is only about $0.47^{\circ}$. Besides, the amplitude and phase measured at both substations also agree with theoretical values very well. This fact thereby verifies the synchronization between this two PMUs. It is worthy to note that there are a lot of different cases tested in our studies and all provide extremely accurate results.

\section{Simulation Example}

\section{PERFORMANCE EVALUATION}

A $345 \mathrm{kV}, 100$ kilometers transposed transmission line is selected to simulate with distributed parameters model. Both ends of the line are replaced by Thévenin's equivalent impedance with an angle different than that of the line impedance (same value as Ref. [5]) The total time of simulation is $T_{10}=200(\mathrm{~m}-\mathrm{sec})$, and the data sampled at sampling rate of $1.92 \mathrm{kHz}(32$ times $60 \mathrm{~Hz})$. For convenient, the fault time are intentionally set at 37.47 milliseconds for all simulation cases. The moving data window contains thirtytwo measured data per cycle, and which are initiated from the simulation start. We first conduct performance evaluation for transmission parameter estimation algorithm and SDFT algorithm, and then fault location index. The error of using the proposed fault location index, $D$, is expressed as a percentage of the total line length, i.e.

$$
\begin{aligned}
\% \text { error } & =\frac{\text { Estimated Location }- \text { Actual Location }}{\text { Total Line Length }} \times 100 \% \\
& =\left(D_{\text {estimated }}-D_{\text {actual }}\right) \times 100 \%
\end{aligned}
$$

The errors between the estimated location and actual location are plotted by percentage on the $\mathrm{Y}$-axis of the figure : all distances are measured from the receiving substation. An error of $-0.1 \%$ indicates that the index determines the fault to be $0.1 \%$ of the total line length closer to the receiving substation than the actual location.

\section{Performance of Line Parameter Estimation Algorithm}

In this paper, we take characteristic impedance and propagation constant of line as line parameters, whose results are illustrated in Fig.6-(a) and (b), to demonstrate the performance of the proposed approach. In this test, we will adopt $\pm 10 \%$ variation of line parameters about the true values as the quantities of uncertainty (same as Ref.[9] ). From Fig.6-(a) and (b), it can be clearly seen that estimated parameters will trace exact parameters very well. The errors of both parameters are remain well within $\pm 0.01 \%$. This parameter estimation algorithm has been demonstrated with field test at $161 \mathrm{kV}$ substation of Taipower system, and the results are very good.

\section{Performance of SDFT method}

First, we show that SDFT could obtain an exact frequency calculation under frequency deviation in a pure sinusoidal waveform. The conventional DFT and the SDFT are utilized to extract phasor and frequency. In Fig.7, we put the emphasis on the frequency variation. The frequency of test signal in Fig. 7 is set at $59.5 \mathrm{~Hz}$. The extract phasor, frequency, phase error, and are illustrated in Fig.7-(a), (b) and (c) respectively. From Fig.7, it is observed that the conventional DFT method gives the worser phasor and frequency estimates. However, the SDFT method could give a stable phasor measurement and an exact frequency identical to the setting value, i.e. $59.5 \mathrm{~Hz}$. We know that if a method can be used in real world, it must take noise into consideration. The frequency of test signal in Fig. 8 is $60 \mathrm{~Hz}$, and we add one percent of white noise into signal. In Fig. 8 , it is clearly seen that SDFT is better than DFT and SDFT ${ }_{357 n}$ is better than SDFT. Fig. 9 shows an another test case. In this test case, we use EMTP to simulate a-phase ground fault. In order for completely reflective to actual system operating situations, Gaussian-type noise (proposed by A. G. Phadke et. al. [14]) will be added into simulated data. The fault location was set to be $D=0.5$. Simulated frequency was intentionally chosen to be $60.5 \mathrm{~Hz}$. From Fig.9, it is observed that the conventional DFT gives the worser phasor and frequency estimates. However, the SDFT method could give a stable phasor measurement and an exact frequency identical to the setting value in EMTP, i.e. $60.5 \mathrm{~Hz}$.

\section{Performance Evaluation for Adaptive Fault Location Index}

\section{Effects of Fault Type and Fault Location}

In order to demonstrate the performance of the proposed fault location index, we have generated a number of test cases using an EMTP. This includes all possible types of fault. Selected results are presented in terms of error in the fault location estimates, and are shown in Fig.10. As Fig. 10 shows, the errors of the proposed index are well within $\pm 0.4 \%$ for most of fault types and fault locations, which means that the accuracy of this index is essentially independent of fault type and fault location. In general, the accuracy of fault events associated with ground faults is better than that of line-to-line short faults (i.e. ungrounded faults). Moreover, it can be 


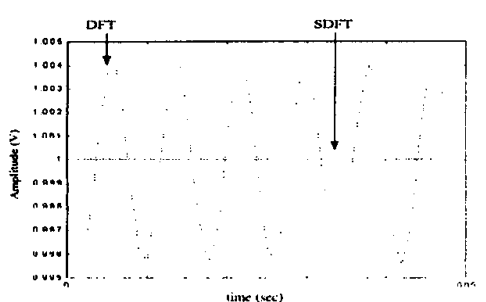

(a) amplitude

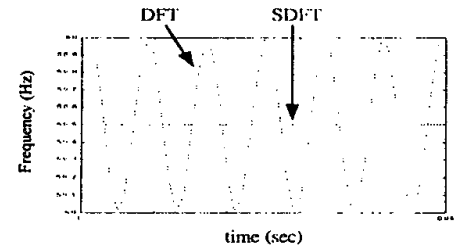

(b) frequency

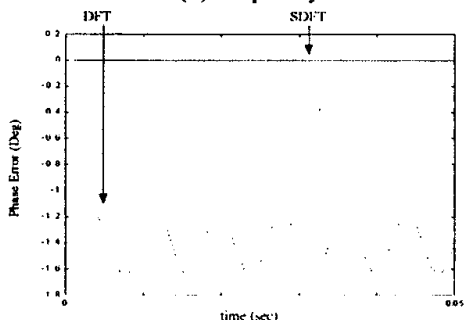

(c) phase error

Fig.7 Test signal: $v(t)=\cos (\omega t)$; simulated frequency $=59.5 \mathrm{~Hz}$; sampling frequency $=960 \mathrm{~Hz}$.
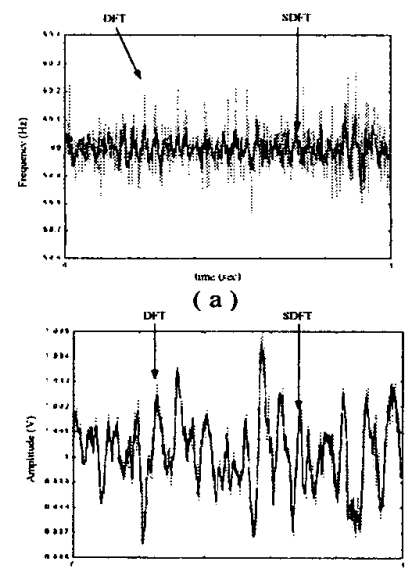

(c)

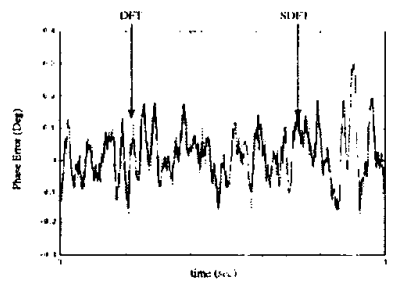

(b)

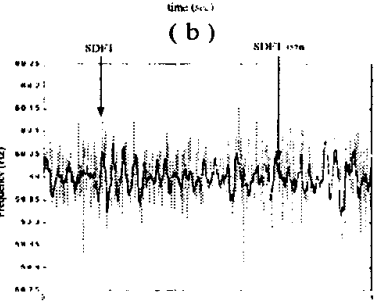

(d)
Fig. 8 test signal: $v(t)=\cos (\omega t)$; simulated frequency 60 $\mathrm{Hz}$; sampling frequency $960 \mathrm{~Hz}$; with $1 \%$ noise.

seen from Fig. 10 that two peak errors of (about $0.417 \%$ and -0.306 $\%$ ) a-b line short faults occurred at 40 and $60 \mathrm{~km}$, respectively. The same situation is in a three-line short fault, and these two larger errors are about $-0.04 \%$ and $0.12 \%$ located in the mentioned positions. Although the proposed index displays the phenomenon mentioned above, the maximum error of the index still remains well within $\pm 0.2 \%$ for most fault events.

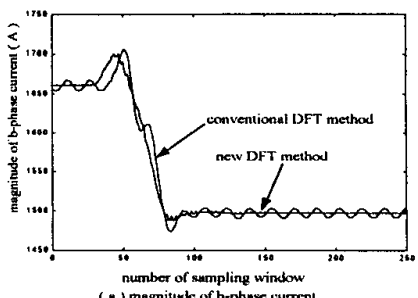

(14) magnitude of b-phasese current

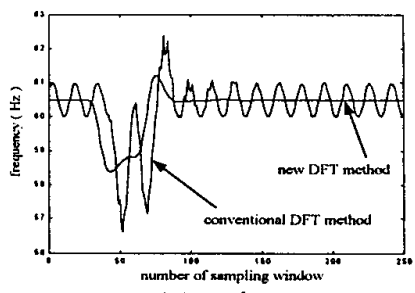

(c) system frequency

Fig.9 Comparison between conventional DFT mettrod freguency and pthsor close-in fundamental frequency under b-pltase ground fault.

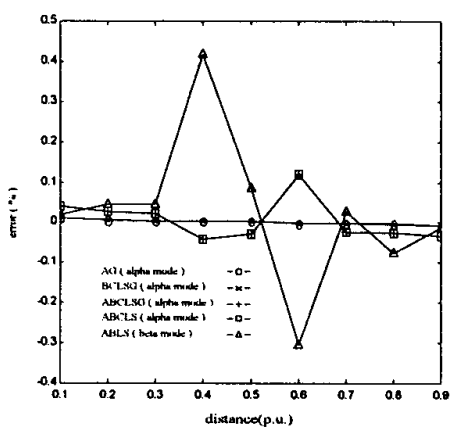

Pig. I 10 Effoct of fault type and frult locetion on measurement

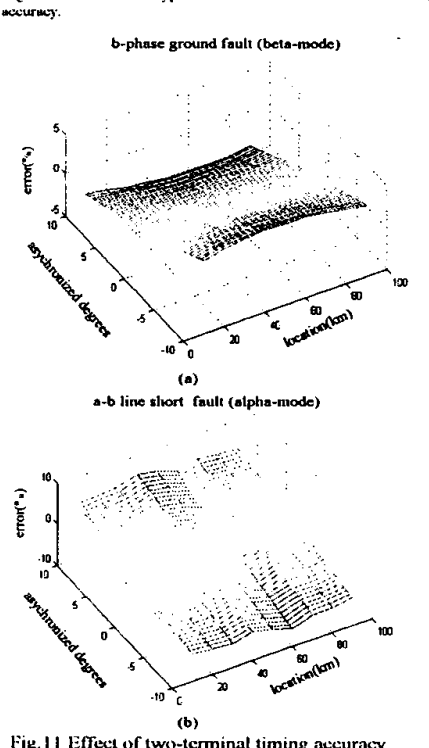

Fig.11 Effect of two-1 for various fault type.

\section{Effects of Two-terminal Timing Accuracy}

Fig.11- (a) and (b), respectively show the errors of single - phase ground fault and double-line short fault due to the data measured at both ends of transmission line asynchronously. In our investigations, it is observed that the synchronization error involved in ungrounded fault cases are much more than that of other fault events. When 


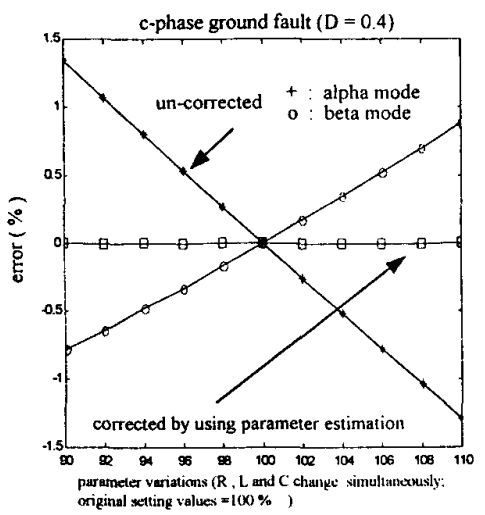

Fig. 12 Performance evaluation of parameter estimation algorithm.

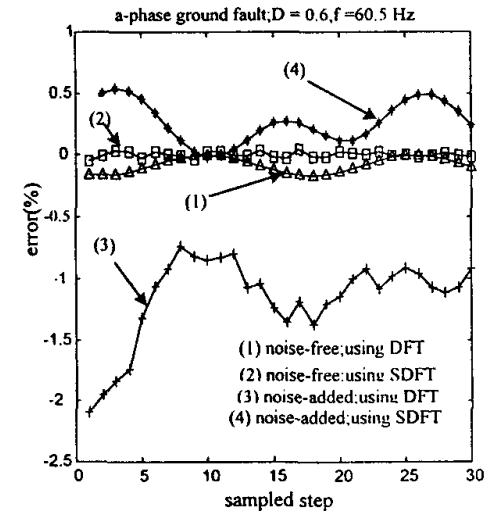

Fig.13 Comparison between DFT and SDFT for a phase ground fault under frequency-floating and system noise consideration.

synchronization error is 10 degree, for example, it will cause up to $4.76 \%$ (minus sign has been neglected) error in the fault location accuracy under the b-phase ground fault cases. Under those ungrounded fault events, e.g. a-b line short, we find that the effect of timing error is more severe on fault location accuracy. These factsare illustrated in Fig.11-(b) and when synchronization error is set at 10 increased up to $-5.66 \%$ under the case of fault location index D $=0.6$ (p.u.).

The synchronization problem can be solved by use of a timing signal such as GPS. In the proposed technique, we have designed an extremely accurate timing device (GSCG). which is equipped in PMU and can be utilized to precisely provide a timing clock signal synchronized with the timing signal issued by GPS satellite. As mentioned in section $\mathrm{V}$, the timing error between two GSCG'S clock DT is able to remain within $1 \mu$ s (equivalent to $0.0216^{\circ}$ ), and its performance has also been demonstrated in field test very well (see Fig.5 shown). In our investigations, it is seen that the synchronization error in the data measured by the PMU based on GSCG can remain well within 1 degree for most of test cases. This fact guarantees that the location accuracy of the proposed index will be hardly affected by the synchronization error.

\section{Effects of Line Parameter Uncertainty}

Usually, line parameters do not vary consistently. or rather they would change vagariously. Fig. 12 shows the error associated with the variation of line parameters for c-phase ground fault. In this test case, the variations of various line model parameters in EMTP are all from $-10 \%$ to $+10 \%$. The fault location is set at $D=0.4$ (p.u.).
From Fig.12, we find that there indeed exists an error in fault location accuracy due to parameter uncertainty. In order to eliminate the error involved with such uncertainty described previously, the proposed line parameter estimation serves the purpose. As Fig.12 shows, when we use the parameter estimation algorithm to correct such parameter uncertainties, the results are almost unaffected by the mentioned problems.

Effects of System Noise and Frequency Fluctuation

Consider the a-phase ground fault case, whose result is shown in Fig.13. In this case, the simulated system frequency and fault location are set to be $60.5 \mathrm{~Hz}$ and 60 kilometers away from receiving end of line, respectively. In this test, Gaussian-type noise has also been added into the raw data generated by EMTP. The measuring error of the SDFT method will achieve well within $0.5 \%$. It is once again found that the performance of the SDFT method is superior to that of conventional DFT method.

\section{CONCLUSION}

In this paper, we propose an adaptive fault location technique. The proposed index does not need any assumption, so it is a very robust index. The parameter estimation algorithm and SDFT method have also been developed in this paper. With the advent of these algorithms the proposed technique can achieve an excellent performance for locating the fault of transmission line. In this study, we have run a great deal of EMTP simulations. These simulation data are utilized to evaluate the performance of the proposed algorithm under the various fault events, un-synchronization errors, system frequency fluctuations, and line-parameters aging effects. Simulation results show that the parameter estimation algorithm can trace parameter variations very well. Hardware errors, system noises, harmonics, and system frequency fluctuation problem have been considered in this paper. A special filtering technique so-called the SDFT method has been developed to solve such problems mentioned above. The performance of the SDFT method for extracting true system frequency and fundamental phasors has been verified. The accuracy of fault location estimation can achieve even up to $99.9 \%$ for many simulated cases. A phasor measurement unit (PMU) with high performance is designed and implemented in the paper. Combining robust fault location index, parameter estimation algorithm, the SDFT method, and the well-designed PMU, the proposed technique will be an adaptive, high performance, and lowcost fault detection/location technique.

\section{References}

[1] T. Takagi, Y. Yamakoshi, J. Baba, K. Uemura, and T. Sakaguchi, "A New Alogrithm of an Accurate Fault Location for EHV/UHV Transmission Lines: Part I - Fourier Transformation Method", IEEE Transactions on Power Apparatus and Systems, Vol. PAS-100, No. 3, March 1981, pp.1316-1323.

[2] T. Takagi, Y. Yamakoshi, J. Baba, K. Uemura, and T. Sakaguchi, "A New Algorithm of an Accurate Fault Location for EHV/UHV Transmission Lines: Part II - Laplace Transform Method", IEEE Transactions on Power Apparatus and Systems, Vol. PAS-101, No. 3, March 1982, pp.564-573.

[3] M. Kezunovi[, J. Mrki D, and B. PeruniciD, "An Accurate Fault Location Algorithm Using Synchronized Sampling", Electric Power Systems Research, Vol. 29, 1994, pp.161-169.

[4] A. A. Girgis, D. G. Hart, and W.L. Peterson, "A New Fault Location Technique For Two- and Three-Terminal Lines", IEEE Transactions on Power Delivery, Vol. 7, No.1, January 1992, pp.98-107.

[5] D. Novosel, D. G. Hart, E. Udren, and J. Garitty, "Unsynchronized Two-Terminal Fault Location Estimation", IEEE Transactions on Power Delivery, Vol. 11, No. 1, January 1996, pp. 130-137. 
[6] A. T. Johns, and S. Jamali, "Accurate Fault Location Technique For Power Transmission Lines", IEE Proceedings, Vol.137, Pt.C, No. 6, November 1990, pp.395-402.

[7] P. K. Aggarwal, D. V. Coury, A. T. Johns, and A. Kalam, "A Practical Approach To Accurate Fault Location on Extra High Voltage Teed Feeders", IEEE Transactions on Power Delivery, Vol. 8, No. 3, July 1992, pp.874-883

[8] D. J. Lawrence, L. Z. Cabeza, and L. T. Hochberg "Development of an Advanced Transmission Line Fault Location System, Part I: Input Transducer Analysis and Requirements", IEEE Transactions on Power Delivery, Vol. 7, No. 4, October 1992, pp. 1963-1971

[9] D. J. Lawrence, L. Z. Cabeza, and L. T. Hochberg, "Development of An Advanced Transmission Line Fault Location System, Part 11: Algorithm Development and Simulation", IEEE Transactions on Power Delivery, Vol. 7, No. 4, October 1992, pp. 1972-1981

[10] B. Lian and M. M. A. Salama "An Overview Of Digital Fault Location Algorithms For Power Transmission Lines Using Transient Waveforms", Electric Power Systems Research, Vol. 29, 1994. pp.1725.

[11] Power Engineering Education Committee and the Power System Relaying Committee of the IEEE Power Engineering Society, "Advancements in Microprocessor Based Protection and Communication", IEEE Tutorial Course, 1997, pp. 63-72.

[12] A. G. Phadke and J. S. Thorp, "Computer Relaying For Power Systems", John Wiley \& Sons, 1988.

[13] H. Y. Li, E. P. Southern, P.A. Crossley, S. Potts, S. D. A. Pickering, B. R. J. Caunce and G. C. Weller, "A New Type of Differential Feeder Protection Relay Using the Global Positioning System for Data Synchronization", IEEE Transaction on Power Delivery, Vol. 12, No. 3, July 1997, pp. 1090-1097.

[14] A. G. Phadke, J. S. Thorp, and K. J. Karimi, "State Estimation with Phasor Measurements", IEEE Transactions on Power Systems, Vol. PWRS-1, No. 1, February 1986, pp. 233-241

[15] J. A. Brandão Faria, and J. H. Briceño Mendez, "Modal Analysis of Untransposed Bilateral Three-Phase Lines - A Perterbation Approach", IEEE Transactions on Power Delivery, Vol.12. No.l, October, 1997, pp. 497-504.

[16] J. Z. Yang and C. W. Liu, "A Smart Method Makes DFT More Precise for Power System Frequency Estimation", In IEEE/PES Winter Meeting, January 1999, pp. 909-913.

Biographies

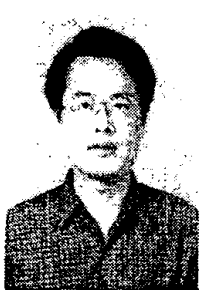

Joe-Air Jiang was born in Tainei, Taiwan, in 1963. He graduated from National Taipei University of Technology in 1983 and received M. S. degree in electrical engineering from National Taiwan University, Taipei, Taiwan in 1990. Since 1990, he has been with Private Kuan-Wu Institute of Technology and Commerce. where he is instructor of electrical engineering. He is currently working on a $\mathrm{Ph} . \mathrm{D}$ research project in power system protection and control, also at National Taiwan University. His area of interest is computer relaying and bio-effects of EM-wave.

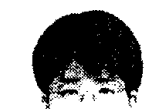

Ying-Hong Lin was born at Taipei, Taiwan, in 1970 He received his B.S. degree in electrical engineering from Taiwan University of Technology in 1995 and is presently working on M.S. degree from National Taiwan University, Taipei, Taiwan. His interested researches are the application of GPS and PMU in power system.

Jun-Zhe Yang was born at Tainan, Taiwan, in 1971. He received his B.S. degree in electrical engineering from Tatung Institute of Technology in 1992 and M.S. degree from National Taiwan University in 1995. He is presently a graduate student in the electrical engineering department, National Taiwan University, Taipei, Taiwan. University of Technology in 1995 and is presently working on Ph.D degree from National Taiwan University, 'Taipei, Taivan.

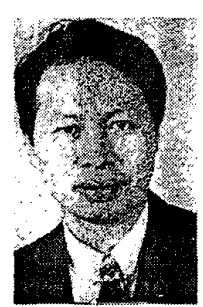

Chih-Wen Liu was born in Taiwan in 1964. He received the B.S. degree in Electrical Engineering from National Taiwan University in 1987, and M.S. and Ph.D. degrees in electrical engineering from Comel University in 1992 and 1994. Since 1994, he has been with National Taiwan University, where he is associate professor of electrical engineering. He is a member of the IEEE and serves as a reviewer for IEEE Transactions on Circuits and Systems, Part I. His main research area is in application of computer technology to power system monitoring, operation, protection and control. His other research interests include GPS tirne transfer and chaotic dynamics and their application to system problems.

Tong-Ming Too was born at Yuan-Lin, Taiwan, in

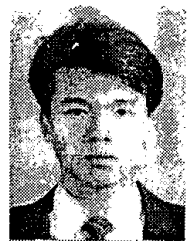
1971. He received his M.S. degree in electrical engineering from Taiwan University in 1998 and is presently working on Mass Transportation Bureau in Taipei, Taiwan. His interested researches are the measurement technique in power system. 